\title{
Singularity Theory and Symplectic Topology
}

\author{
Alexander B. Givental
}

It is mandatory to begin a lecture with something transparent for any undergraduate math major. Preparing this talk I tried to recall what I myself was able to understand twenty years ago when I first came to V. Arnold's seminar on singularity theory. My choice of the subject area was due to an advice of a senior student who explained that singularity theory is worth studying because it is situated on the intersection of virtually all branches of mathematics. In this lecture, I will try to show that the advice was not so bad indeed.

Singularity theory [2]. The most of the applications of singularity theory are based on the theorem that the critical point of the function $x^{3}$ decays into a local maximum and a local minimum when the function is perturbed to $x^{3}-\lambda x$. Therefore a solid calculus course is among the prerequisites for students specializing in singularity theory.

The number of non-degenerate critical points to which $f=x^{\mu+1}$ decays under a generic perturbation $f_{\lambda}$ can be computed algebraicly as the dimension of the local algebra

$$
Q:=\mathbb{C}[x] /(\partial f / \partial x)
$$

spanned by the monomials $1, x, x^{2}, \ldots, x^{\mu-1}$ which are to be multiplied modulo $x^{\mu}=0$. Therefore the abstract algebra should be learned as early as possible.

It takes a great deal of complex analysis to prove the general multiplicity formula $\mu=\operatorname{dim} Q$ based on the property of the residue pairing

$$
\langle\varphi, \psi\rangle:=\operatorname{Res}_{\infty} \frac{\varphi(x) \psi(x) d x}{\partial f_{\lambda} / \partial x}
$$

to define a non-degenerate symmetric bilinear form on the algebra $Q_{\lambda}:=$ $\mathbb{C}[x] /\left(\partial f_{\lambda} / \partial x\right)$ of functions on the critical set. 
Furthermore, all perturbation patterns of the critical point $f$ are contained in the miniversal deformation obtained by adding to $f$ a general linear combination of the monomials spanning the local algebra:

$$
f_{\lambda}(x)=x^{\mu+1}+\lambda_{1} x^{\mu-1}+\lambda_{2} x^{\mu-2}+\ldots+\lambda_{\mu-1} x+\lambda_{\mu} .
$$

In symplectic geometry, a family of functions generates a lagrangian submanifold $L$ in the cotangent bundle $T^{*} \Lambda$ of the parameter space. It is parametrized by critical points $x_{c r i t}$ of the functions from the family:

$$
L:=\left\{(p, \lambda) \mid \exists x_{\text {crit }}: p=d_{\lambda} f_{\lambda}\left(x_{\text {crit }}\right)\right\} .
$$

The lagrangian submanifolds generated by miniversal deformations of isolated critical points provide normal forms for singularities of wave fronts and caustics arising in geometrical optics and classical mechanics.

In the wave optics, one of the approaches is based on studying oscillating integrals of the form

$$
I_{\hbar}(\lambda):=\int_{\Gamma} \exp \left(f_{\lambda}(x) / \hbar\right) d x
$$

as generalized Airy functions of the parameters $\lambda$ of the miniversal deformation. It is the stationary phase approximation

$$
\int \exp \left(f_{\lambda}(x)\right) d x \sim \frac{e^{f\left(x_{c r i t}\right) / \hbar}}{\sqrt{\operatorname{Hess}_{x_{c r i t}} f_{\lambda}}}
$$

to the oscillating integral that links wave theory with geometrical optics in the "short wave limit" $\hbar \rightarrow 0$.

Similarly to the classical Airy functions, the oscillating integrals can be described via solutions to Picard-Fuchs linear differential equations. The monodromy of such solutions around the discriminant in the complex parameter space $\Lambda$ is determined by the monodromy of the integration cycles $\Gamma$ constructed by tools of Morse theory for the real functions $\operatorname{Re} f_{\lambda}$. Thus singularity theory makes use of algebraic and differential topology as well.

The monodromy groups turn out to be reflection groups, and the theory - related to Dynkin diagrams, the ADE-classification of simple Lie algebras, representations of binary groups of regular polyhedra, the theory of automorphic functions, the Chevalley theorem for complex cristallographic 
groups and many other interesting topics a beginning math student is anxious to learn.

Symplectic topology. After 1982 the subject of Arnold's seminar began to drift toward the direction quite remote from singularity theory. The change was caused by Conley - Zehnder's proof [4] for symplectic tori of the Arnold fixed point conjecture [1] saying that hamiltonian transformations on compact symplectic manifolds must have fixed points.

The proof, based on fairly fresh ideas, inspired several new applications, and in particular - Ya. Eliashberg's "existence theorem of symplectic topology" [7]: symplectomorphism groups are $C^{0}$-closed in the groups of volume-preserving diffeomorphisms. In $1984 \mathrm{M}$. Gromov [14] proposed to construct topological invariants of symplectic manifolds by studying holomorphic curves in the spirit of classical enumerative algebraic geometry.

Consider the complex projective space $\mathbb{C} P^{n}$ as a model example of a real $2 n$-dimensional manifold with the imaginary part of the Fubbini Kähler form on the role of the symplectic structure. One can think of the cohomology algebra of $\mathbb{C} P^{n}$ in Poincaré-dual terms of cycles $1, p, p^{2}, \ldots, p^{n-1}$ representing the fundamental class, hyperplane section, codimension 2 subspace, etc. The algebra is provided with the non-degenerate Poincaré intersection pairing

$$
\langle\varphi, \psi\rangle=\frac{1}{2 \pi i} \oint \varphi(p) \psi(p) \frac{d p}{p^{n}}
$$

The only obvious topological invariant of a symplectic structure is the cohomology class of the symplectic differential 2-form in the cohomology algebra.

According to M. Gromov, a handful of non-trivial symplectic invariants can be constructed by counting compact holomorphic curves in $\mathbb{C} P^{n}$ of given genus and degree passing through given generic cycles. For example, the series

$$
F(t)=\sum_{k=0}^{\infty} \frac{1}{k !}(t, t, \ldots, t)
$$

where $(t, \ldots, t)$ means the number of rational curves passing through $k$ generic cycles representing the same general cohomology class $t=t_{0}+t_{1} p+t_{2} p^{2}+$ $\ldots+t_{n-1} p^{n-1}$ encodes information about genus 0 invariants.

According to E. Witten [18], the invariants are not independent (to regrets of symplectic topologists and benefits of algebraic geometers) and obey some universal identities. For example, the 3-rd partial derivatives $F_{\alpha \beta \gamma}$ of $F$, 
considered as a non-linear function on the cohomology space $H$, are structural constants $\left\langle p^{\alpha} o_{t} p^{\beta}, p^{\gamma}\right\rangle$ of a commutative associative multiplication $o_{t}$ on the tangent space $T_{t} H$ called the quantum deformation of the classical cupproduct.

Moreover, the system of linear differential equations

$$
\hbar \partial_{\alpha} \vec{I}=\left(p^{\alpha}\right) \circ_{t} \vec{I}
$$

to be solved for a cohomology-valued vector function on $H$ is consistent for any non-zero value of the parameter $\hbar$. This integrability condition and the associativity of the quantum cup-product represent the universal identities among the genus 0 Gromov-Witten invariants of compact symplectic manifolds.

Furthermore, the asymptotic behavior of solutions $I_{\hbar}(t)$ to the above differential equations as $\hbar \rightarrow 0$ can be described by the "stationary phase anzatz"

$$
I_{\hbar} \sim \sum_{i} \operatorname{Coeff}_{i} \frac{e^{u_{i} / \hbar}}{\sqrt{\Delta_{i}}}
$$

where $u_{i}$ and $\Delta_{i}$ are functions of $t$. They describe the common eigenvalues $\partial_{\alpha} u_{i}$ of the commuting quantum multiplication operators $p^{\alpha} o_{t}$ and the Poincaré intersection paring in the basis of eigenvectors respectively.

In particular, the quantum cohomology algebra can be considered as the algebra of functions on a subvariety $L$ in the cotangent bundle space $T^{*} H$, the variety $L$ is necessarily lagrangian since its branches over $H$ are locally represented by the differentials $d u_{i}$, and the Poincaré intersection pairing on $T_{t} H$ is given by the "residue anzatz"

$$
\langle\varphi, \psi\rangle=\sum_{p_{i} \in L \cap T_{t}^{*} H} \frac{\varphi\left(p_{i}\right) \psi\left(p_{i}\right)}{\Delta_{i}} .
$$

Quantum field theory. The parallel between the structure of GromovWitten invariants in symplectic topology and the data arising in the theory of critical points of holomorphic functions can be formalized in the axiomatic language of 2-dimensional conformal quantum field theory. In this theory, the space-time is represented by a Riemannian surface $\Sigma$, the fields $\phi$ are maps from the surface to a suitable target space, and a theory is specified by a choice of the action functional $\mathcal{S}\{\phi\}=\int_{\Sigma} \mathcal{L}(\phi, d \phi, \ldots)$ of the fields 
which may depend on the conformal class of the metric on the space-time. Correlators of the quantum field theory are supposed to be defined by the Feynman path integrals over the space of all fields and Riemannian metrics

$$
(a, b, \ldots, c)=\int a(\phi) b(\phi) \ldots c(\phi) \exp (i \mathcal{S}\{\phi\})
$$

where the observables $a, b, \ldots, c$ are some functionals of the fields and may also depend on the conformal class of the metric on $\Sigma$.

In the axiomatic approach to the quantum field theory one assumes that the functional integration over the space of all fields (which is hard to make sense of) has been already performed to yield a closed differential form on the moduli space of conformal structures. It remains only to integrate this form over a cycle in the moduli space.

The moduli spaces in question usually denoted $\overline{\mathcal{M}}_{g, k}$ are compactified Deligne-Mumford spaces of so called stable genus $g$ compact complex curves with $k$ marked points. One can invent many meaningful constructions of cycles in these spaces. Each such construction generates a correlator to be defined in any 2-dimensional quantum field theory. The relations between the homology classes of the cycles represent therefore the universal identities between the correlators. Thus the system of axioms is implicitly encoded by the topology of all the Deligne-Mumford spaces. For example, the simplest of the Deligne-Mumford spaces is the sphere $\overline{\mathcal{M}}_{0,4}$ of values of the cross-ratio $z$ among 4 marked points on $\mathbb{C} P^{1}$. The forbidden values $z=0,1, \infty$ of the cross-ratio correspond to the 3 ways of distributing the 4 points in pairs among the two components of the reducible rational curve which consists of two intersecting straight lines. The associativity of the quantum cup-product originates from the simple fact that the points $z=0,1$, and $\infty$ represent the same homology class of $\overline{\mathcal{M}}_{0,4}$.

Algebraic topology of other Deligne-Mumford spaces is fairly sophisticated and is understood well only in genus 0 and 1 (see [10]). Nevertheless one can show that for any compact symplectic manifold the Gromov-Witten invariants, which correspond to cycles in Deligne-Mumford spaces by the very definition, indeed satisfy the axioms of the 2-dimensional quantum field theory. This fact has been rigorously established by several groups of authors [9] which completed the project started by M. Kontsevich [15] in 1994.

To the contrary, in singularity theory the possibility to associate a 2dimensional quantum field theory to any isolated critical point remains un- 
certain. However the fact that the axioms for genus 0 correlators alone hold true (see [6]) turns out to be equivalent to K.Saito's approach [17] to complex oscillating integrals via so called primitive forms and flat coordinates which has been known in singularity theory since 1982 .

The mirror conjecture. It should have been a great surprise for participants of Arnold's seminar to learn that their research in the two remote directions can be unified by a single system of axioms. Yet this fact does not indicate any direct relationship between singularity theory and symplectic topology. The idea that such a relationship might exist came from string theory as The Mirror Conjecture. The idea suggests that not only the axioms are the same, but also the realization of the axioms by Gromov-Witten invariants of a particular symplectic manifold might coincide with a particular realization of singularity theory type.

Attempting to make the idea more precise, we may conjecture (see [11]) that to a $2 n$-dimensional compact symplectic manifold $X$ one can naturally associate the mirror partner of $X$, which consists of a family $Y_{\lambda}$ of $n$-dimensional complex algebraic manifolds, a family $f_{\lambda}$ of holomorphic functions $Y_{\lambda} \rightarrow \mathbb{C}$ and a family $\omega_{\lambda}$ of holomorphic volume forms on $Y_{\lambda}$, in such a way that the structure on the parameter space $\Lambda$ defined in terms of critical points, residue pairings and complex oscillating integrals

$$
\int_{\Gamma \subset Y_{\lambda}} \exp \left(f_{\lambda} / \hbar\right) \omega_{\lambda}
$$

is isomorphic to the structure defined by the genus 0 Gromov-Witten invariants of $X$ on the cohomology space $H$. This formulation can be supported by several classes of examples. Yet the mirror conjecture resembles a universal "physical" principle (like the Energy Conservation Law which is to be modified every time it conflicts with experimental data) rather than a precise mathematical conjecture which is to be proved or disproved.

As some examples suggest, in the Calabi-Yau case, when $X$ is Kähler and admits holomorphic volume forms, the mirror manifolds $Y_{\lambda}$ should be compact (so that $f_{\lambda}=$ const) but still carry the holomorphic volume form $\omega_{\lambda}$. Thus the oscillating integrals degenerate into the periods $\int_{\gamma \subset Y_{\lambda}} \omega_{\lambda}$ distinguishing complex structures on the - also Calabi-Yau - manifolds $Y_{\lambda}$. The relationship between $X$ and $Y$ is symmetric: Gromov-Witten invariants of $Y$ are to be equivalent to invariants of the complex structures on $X$. This 
"classical" case of the conjecture gave the name to the mirror symmetry phenomenon. As it is clear now, the first non-trivial example of mirror symmetry — for some $K 3$ surfaces — has been known in singularity theory since 1974 under the name "Arnold's strange duality" (see [5]).

Examples. We outline below two applications of the mirror correspondence between singularity theory and symplectic topology.

Consider the complex oscillating integral

$$
I_{\hbar}(t)=\int_{\Gamma \subset\left\{x_{0} \ldots x_{n}=e^{t}\right\}} e^{\left(x_{0}+\ldots+x_{n}\right) / \hbar} \frac{d x_{0} \wedge \ldots \wedge d x_{n}}{d\left(x_{0} \ldots x_{n}\right)} .
$$

It satisfies the differential equation $(\hbar d / d t)^{n+1} I=e^{t} I$ with the symbol $p^{n+1}=$ $e^{t}$. This identifies the integral as the mirror partner of $\mathbb{C} P^{n}$. Indeed, the fact that there is exactly one straight line passing through two distinct points in $\mathbb{C} P^{n}$ can be encoded (see [8]) by the relation $p^{n+1}=e^{t}$ in the quantum cohomology algebra of $\mathbb{C} P^{n}$ where $t$ is the coordinate on $H^{2}\left(\mathbb{C} P^{n}\right)$. This fact is known to determine all other genus 0 Gromov-Witten invariants of $\mathbb{C} P^{n}$ via the universal axioms (see [16]).

Consider now a non-singular degree $n+1$ hypersurface $X$ in $\mathbb{C} P^{n}$. It is an example of a Calabi-Yau manifold, and the genus 0 Gromov-Witten invariants of $X$ depend on infinitely many "independent" numbers $m_{d}, d=$ $1,2,3, \ldots$, accountable for degree $d$ rational curves in $X$.

Theorem [12]. Genus 0 Gromov-Witten invariants of $X$ are encoded by the degenerate "oscillating integral"

$$
\int_{\gamma \subset\left\{x_{0} \ldots x_{n}=e^{t}, x_{0}+\ldots+x_{n}=1\right\}} \frac{d x_{0} \wedge \ldots \wedge d x_{n}}{d\left(x_{0} \ldots x_{n}\right) \wedge d\left(x_{0}+\ldots+x_{n}\right)} .
$$

For $n=4$ the theorem allows one to confirm the predictions $m_{1}=$ $2875, m_{2}=609250, m_{3}=317206375, m_{4}=242467530000, \ldots$ for the numbers of degree $d$ rational curves on quintic Calabi-Yau 3-folds made in 1991 by Candelas, de la Ossa, Green and Parkes [3] on the basis of the mirror conjecture. The result can be extended to Fano and Calabi-Yau complete intersections in projective spaces and more general toric manifolds. Also, the theorem motivates the quantum Lefschetz hyperplane section conjecture which identifies the interior integral in the double integral

$$
\int_{Y} e^{f_{\lambda} / \hbar} \omega_{\lambda}=\int e^{\tau / \hbar} d \tau \int_{f_{\lambda}=\tau} \omega_{\lambda} / d f_{\lambda}
$$


as the mirror partner of the anti-canonical hypersurface in a Fano manifold $X$ in terms of the mirror partner $\left(Y_{\lambda}, f_{\lambda}, \omega_{\lambda}\right)$ of $X$.

In the second example we express the generating function $G: H \rightarrow \mathbb{C}$,

$$
G(t)=\sum_{k=0}^{\infty} \frac{1}{k !}[t, t, \ldots, t],
$$

for genus 1 Gromov-Witten invariants in terms of genus 0 Gromov-Witten invariants of $X$ encoded by the oscillating integral $\int \exp \left(f_{t} / \hbar\right) \omega_{t}$. Take the critical values $u_{i}, i=1, \ldots, \operatorname{dim} H$, of the Morse function $f_{t}$ for local coordinates near a generic point $t \in H$. Let $\Delta_{i}$ denote the Hessian of $f_{t}$ at the critical point with respect to $\omega_{t}$. Let $R_{i}$ denote another characteristic of the critical point obtained from the stationary phase asymptotic expansion near this critical point:

$$
\hbar \frac{\partial}{\partial u_{i}} \int e^{f_{t(u)} / \hbar} \omega_{t(u)}=\text { const } \hbar^{(\operatorname{dim} Y) / 2} e^{u_{i} / \hbar}\left[1+R_{i} \hbar+o(\hbar)\right] .
$$

\section{Conjecture.}

$$
d G=\frac{1}{48} \sum_{i} \frac{d \Delta_{i}}{\Delta_{i}}+\frac{1}{2} \sum_{i} R_{i} d u_{i} .
$$

The conjecture is supported by a corresponding theorem [13] applicable to certain class of toric manifolds and toric complete intersections. Also, it suggests how to extend to genus 1 the axiomatic genus 0 quantum 2-dimensional field theory associated in singularity theory to any isolated critical point.

\section{References}

[1] V.I. Arnold. Sur un propriété topologique des applications canoniques de la mecanique classique. C. R. Acad. Sci. Paris 261 (1965), 3719 - 3722.

[2] V. Arnold, A. Varchenko, S. Gusein-Zade. Singularities of differential maps. v. 1, Birkhäuser, Basel, 1985; v.2, Birkhäuser, Basel, 1988.

[3] P. Candelas, X. C. de la Ossa, P. S. Green, L. Parkes. A pair of Calabi-Yau manifolds as an exactly soluble superconformal theory. Nucl. Phys. B 359 (1991), 21 - 74.

[4] C. Conley, E. Zehnder. The Birkhoff - Lewis fixed point theorem and a conjecture of V. I. Arnold. Invent. Math. 73 (1983), 33 - 49. 
[5] I. Dolgachev, V. Nikulin. Exceptional singularities of V. I. Arnold and K3-surfaces. All-Union Topology Conference, Abstract of Papers, Minsk, 1977 (in Russian). H. Pinkham. Singularités exceptionelles, la dualité étrange d'Arnold et le surfaces K3. C. R. Acad. Sci. Paris, Sér. I Math. 284 (1977), 615 - 618.

[6] B. Dubrovin. Geometry of $2 D$ topological field theories. In: Springer Lecture Notes in Math., 1620 (1996), 120 - 348.

[7] Ya. Eliashberg. The wave fronts structure theorem and its applications to symplectic topology. Funct. Anal. Appl. 3 (1987), 65-72.

[8] A. Floer. Symplectic fixed points and holomorphic spheres. Commun. Math. Phys. 120 (1989), $575-611$.

[9] K. Fukaya, K. Ono. Arnold conjecture and Gromov - Witten invariant. Preprint, 1996. Y. Ruan. Virtual neighborhoods and pseudo-holomorphic curves. Preprint, alggeom/9611021. J. Li, G. Tian. Virtual moduli cycles and Gromov - Witten invariants in general symplectic manifolds. Preprint, alg-geom/9608032. K. Behrend, B. Fantechi. The intrinsic normal cone. Invent. Math. 128 (1997), 45 - 88., H. Hofer, D. Salamon. Rational Floer homology and the general Arnold conjecture. In preparation.

[10] E. Getzler. Intersection theory on $\mathcal{M}_{1,4}$ and elliptic Gromov-Witten invariants. Preprint, alg-geom/9612004.

[11] A. Givental. Homological geometry and mirror symmetry. In: Proceedings of the International Congress of Mathematicians, 1994, Zürich, Birkhäuser, Basel, 1995, $472-480$.

[12] A. Givental. Equivariant Gromov - Witten invariants. Intern. Math. Res. Notices, 1996, No. 13, 1 - 63. A. Givental. A mirror theorem for toric complete intersections. alg-geom/9701016.

[13] A. Givental. Elliptic Gromov-Witten invariants and the generalized mirror conjecture. In preparation.

[14] M. Gromov. Pseudo-holomorphic curves in almost complex manifolds. Invent. Math. 82:2 (1985), $307-347$.

[15] M. Kontsevich. Enumeration of rational curves via toric actions. In: The moduli space of curves, R. Dijkgraaf, C. Faber, van der Geer (eds.), Progress in Math. 129, Birkhäuser, 1995, pp. $335-368$.

[16] M. Kontsevich, Yu. Manin. Gromov-Witten classes, quantum cohomology and enumerative geometry. Commun. Math. Phys. 164 (1994), 525 - 562.

[17] K. Saito. On periods of primitive integrals, I. Preprint 412, Res. Inst. Math. Sci., 1982.

[18] E. Witten. Two-dimensional gravity and intersection theory on moduli space. Surveys in Differential Geometry 1 (1991), 243 - 310. 